ISSN 2447-9071

doi https://doi.org/10.36414/rbmc.v7i17.73

Contato para correspondência: Hermínio Maurício da Rocha Sobrinho

E-mail:

herminio.sobrinho@gmail.com

Conflito de interesse: Não

Financiamento: Recursos próprios

Recebido: 04/12/2020

Aprovado: $27 / 02 / 2021$

\section{Diagnóstico e acompanhamento evolutivo dos meningiomas através da ressonância magnética}

\section{Diagnosis and evolutionary follow-up of meningiomas through magnetic resonance}

Raphael da Silva Fonseca Pereira', Cristiene Costa Carneiro', Valéria Bernadete Leite Quixabeira' ${ }^{1}$ Hermínio Maurício da Rocha Sobrinho ${ }^{1,2}$

\author{
1 Pontifícia Universidade Católica de Goiás - PUC Goiás \\ 2 Universidade Estadual de Goiás - UEG
}

\section{Resumo}

Os meningiomas são tumores benignos intracranianos de evolução lenta que se originam nas meninges. Por serem assintomáticos, a maioria desses tumores, geralmente, são de difíiil identificação. Entretanto, se não diagnosticados, podem comprimir vasos sanguíneos cerebrais levando ao óbito. $A$ Ressonância Magnética (RM)éummétodo de imagem vital parao diagnósticoecaracterização desses tumores, particularmente importante para seestabelecerum plano de tratamento oncológico. Oobjetivo desteestudo foidescrever a importância da RM nodiagnóstico e acompanhamento evolutivo dos meningiomas ena caracterização tissular, correlacionando os achados IMAG enológicos com análises histológicas do tumor. Este estudo constitui uma revisão bibliográfica narrativa. Os meningiomas são, geralmente, isointensos em T1 e T2, com variações ligadas à textura mais ou menos compacta ou hidratada do tecido, associados à presença de cistos, calcificações ou metaplasia adiposa. Podem provocar hiperostose, levando ao espessamento do osso afetado. Emimagens ponderadas em T1, pós contraste, os meningiomas, geralmente, aparecem com hipersinal homogêneo. A RM contribui, de formapromissora, para a deteç̧ãoe caracterização dos meningiomas fornecendo dados prognósticos para oseu tratamento. Um diagnóstico preciso auxilia no acompanhamento evolutivo e pós cirúrgico. Em um período de 24 horas pós cirúrgico, a RMécapaz de identificar resíduos tumorais e recorrências tardias, comuns entre 10 a 20 anos depois da ressecção total do tumor.

Palavras-Chave: Meningiomas; Diagnóstico; Ressonância Magnética.

\begin{abstract}
Meningiomas are benign, slowly evolving intracranial tumors that originate in meninges. As they are asymptomatic, most of these tumors are generally difficult to identify. However, if not diagnosed, they can compress brain blood vessels leading to death. Magnetic resonance imaging (MRI) is a vital imaging method for the diagnosis and characterization of these tumors, which is particularly important for establishing a cancer treatment plan. The objective of this study was to describe the importance of MRI in the diagnosis and evolutionary monitoring of meningiomas and in tissue characterization, correlating the imaging findings with histological analysis of the tumor. This study constitutes a narrative bibliographic review. Meningiomas are generally isointense in $T 1$ and $T 2$, with variations linked to the more or less compact or hydrated texture of the tissue, associated with the presence of cysts, calcifications, or adipose metaplasia. They can cause hyperostosis, leading to thickening of the affected bone. In post-contrast T1-weighted images, meningiomas usually appear with homogeneous hypersignal. MRI promises a promising way for the detection and characterization of meningiomas, providing prognostic data for their treatment. An accurate diagnosis helps in the evolutionary and post-surgical follow-up. In a period of 24 hours after surgery, MRl is able to identify tumor residues and late recurrences, common between 10 to 20 years after the total tumor resection.

Keywords: Meningiomas; Diagnosis; Magnetic Resonance Imaging.
\end{abstract}




\section{Introdução}

Desde a pré-história são descritos conhecimentos anatômicos, tendo em vista que fósseis de crânios humanos perfurados foram localizados, datados de mais ou menos três mil anos a.C. Dentre várias obras anatômicas, destaca-se a coleção de Hipócrates (600 - 300 a.C.) e contribuições das descrições ilustradas por Aristóteles. A escola de Alexandria, no Egito, caracterizou-se como a maior instituição científica da antiguidade clássica, onde a anatomia foi descrita pela primeira vez como uma disciplina, sendo que as primeiras dissecações de animais e humanos foram realizadas por Herófilo de Calcedônia, que identificou o cérebro como o principal órgão do Sistema Nervoso Central (SNC), e por Erasítrato'.

Desde o conhecimento anatômico, novas conquistas foram descritas ao longo da história da medicina. Alterações observadas puderam ajudar na identificação de disfunções fisiológicas, e contribuíram para o aumento do conhecimento na área da saúde. Atualmente a tecnologia tem influenciado bastante no diagnóstico das diversas morbidades conhecidas ${ }^{1,2}$.

Os meningiomas são tumores benignos que foram descritos por Richard Bright em 1831 como sendo tumores originados a partir da membrana aracnóidea. Cleland em 1864 sugeriu sua origem dos corpúsculos ou granulações de Pacchioni e Schmidt. Cushing em 1922 atribuiu definitivamente sua origem a partir de células aracnóideas das vilosidades e das membranas aracnóideas. Essa neoplasia pode migrar para tecidos adjacentes, como dura-máter, espaço vascular e todo o crânio ${ }^{3,4}$.

Estes tumores desenvolvem-se preferencialmente na convexidade cerebral, tenda cerebelar, sulcos olfatórios e asa menor do esfenoide. Acometem o SNC, com maior prevalência em mulheres após os cinquenta anos de idade e, normalmente, de forma assintomática. Os casos sintomáticos podem ocorrer devido constrição, oclusão e/ou propagação do tumor em estruturas fundamentais para a fisiologia do corpo humano5,6. O curso da doença pode variar bastante, devido sua evolução lenta, podendo variar entre 15 dias e 7 anos $^{3}$.

Sua etiologia é desconhecida, porém sabe-se de atributos causais, como: fator genético (mutação no gene NF2 e deleção no cromossomo 22q), efeitos tardios pelo contato com elementos radioativos, irritação ou infecção crônica das meninges, hematoma subdural e traumatismos cranianos podem induzir o desenvolvimento deste tumor ${ }^{5-7}$.

Em relação aos métodos para o diagnóstico do meningioma, além dos indícios de alterações hematológicas, o diagnóstico por imagem revela com grande precisão a região acometida e possíveis metástases que, apesar de serem raras, apresentam uma incidência por volta de 1:1000 casos. Os prin- cipais sítios de acometimento metastático dos meningiomas são: pulmões, fígado, linfonodos cervicais, ossos longos, pelve, crânio, pleura, vértebras e $S N C^{2,8}$.

A RM é essencial no diagnóstico de neoplasias do SNC, principalmente no planejamento de intervenções cirúrgicas. Essa técnica, além das vantagens de não utilizar radiações ionizantes, é fundamental no diagnóstico, caracterização tecidual (consistência do tumor) e correlação das análises de imagens com os subtipos histológicos do tumor para análise do prognóstico da doença. A análise da consistência do tumor pela RM pode colaborar para o planejamento das ressecções cirúrgicas mais adequadas do tumor e até mesmo uma substituição da dura-máter subjacente em tratamentos de casos avançados ${ }^{2,9}$.

Desta forma, o objetivo do presente estudo foi descrever a importância e benefícios da RM no diagnóstico e acompanhamento evolutivo dos meningiomas.

\section{Métodos}

O presente estudo trata-se de uma revisão bibliográfica narrativa para a qual foram realizadas buscas de estudos nas plataformas eletrônicas de dados: National Center of Biotechnology Information US (PubMed), Portal de Periódicos da Capes, SciELO e Google Acadêmico. O recorte temporal da pesquisa foi o período de 1993 ao ano de 2020. Para a pesquisa foram utilizados os seguintes Descritores em Ciências da Saúde (DeCS) ou palavras-chave, de forma isolada e combinada, nos idiomas português e inglês: Ressonância Magnética, Meningiomas, Diagnóstico, Exames por Imagem, Magnetic Resonance Imaging, Imaging Exams.

Foram incluídos neste estudo artigos pulicados dentro do período temporal estabelecido, artigos com texto disponível na íntegra e estudos que apresentavam relação com os objetivos propostos. Foram excluídos deste estudo os artigos duplicados, artigos com textos completos não disponíveis, estudos que não apresentavam relação com os objetivos propostos e trabalhos publicados fora do período temporal determinado. Um total de 158 artigos foram encontrados nas bases de dados utilizadas, deste total foram selecionados 33 artigos que apresentavam conteúdos relacionados aos objetivos deste estudo.

\section{Aspectos gerais dos meningiomas}

Os meningiomas são tumores benignos na maioria dos casos, entretanto, há ocorrência de casos anaplásicos com prognósticos ruins que ocorrem em menos de $5 \%$ dos meningiomas ${ }^{10}$.

Segundo a Organizção Mundial da Saúde (OMS)10, os meningiomas podem ser classificados em grau 1, 2 e 3 . Essa 
classificação está ligada a evidências histológicas associadas também à clínica dos pacientes. Nos meningiomas de grau 1 , normalmente de curso benigno, pode se encontrar uma variedade de subtipos como por exemplo: meningotelial ou sincicial, fibroso ou fibroblástico, transicional, psamomatoso, angiomatoso, microcístico, secretor, rico em linfócitos e plasmócitos, e metaplásico. Nos meningiomas grau 2, são encontrados tumores atípicos com células de atividade mitótica aumentada, aumento do número de núcleos das células, aumento da relação núcleo-citoplasmática com nucléolos ressaltados, arquitetura difusa com presença de necrose. $\mathrm{O}$ grau 3 desses tumores são anaplásicos, caracterizados por elevada taxa de atividade mitótica ( $\geq 20 / 10$ em campos visuais de maior aumento), padrão histológico sem distinção pela imunohistoquímica, e alterações genéticas como a deleção do cromossomo 22q e do gene NF2. Apesar dessas diferentes classificações, qualquer meningioma pode ter evolução atípica ou anaplásica ${ }^{10,11}$.

Os tumores anaplásicos apresentam elevado potencial metastático, conforme demonstrado em um estudo no qual pacientes entre 33 e 91 anos de idade, do sexo masculino, apresentavam meningiomas anaplásicos com alterações invasivas nos tecidos ósseos, cerebrais e muscular ${ }^{10}$.

A RM define, operacionalmente, a descoberta tumoral. Os meningiomas são isointensos em relação ao cérebro em imagens registradas em $\mathrm{T} 1$ e $\mathrm{T} 2$. Cerca de 10 a $15 \%$ dos meningiomas tem aparência atípica, simulando metástases ou gliomas malignos. Atualmente, trata-se do melhor exame para diagnóstico e acompanhamento evolutivo dos meningiomas $s^{5,7}$.

A celularidade do tumor, o conteúdo de água e o conteúdo fibroso são considerados os principais determinantes das diferentes intensidades de sinal dos vários subtipos de meningiomas ${ }^{12}$.

Um estudo evidenciou em lâminas histopatológicas obtidas a partir da medula óssea de crânio, uma cavitação com acúmulo de sangue e infiltração tumoral, levando ao aumento da vascularização que não é compensada pela vasodilatação. Isso pode estar relacionado à hiperintensidade apresentada em T2 presumindo uma congestão no osso. A hiperostose foi evidenciada a partir da identificação de uma área mais espessa que o crânio em um sítio diferente do habitual, sendo um achado do seio paranasal adjacente ao meningioma ${ }^{13}$. $\mathrm{O}$ quadro 1 evidencia os principais aspectos e características dos meningiomas.
Quadro 1. Principais aspectos e características clínicolaboratoriais e imagenológicas dos meningiomas em pacientes adultos e pediátricos.

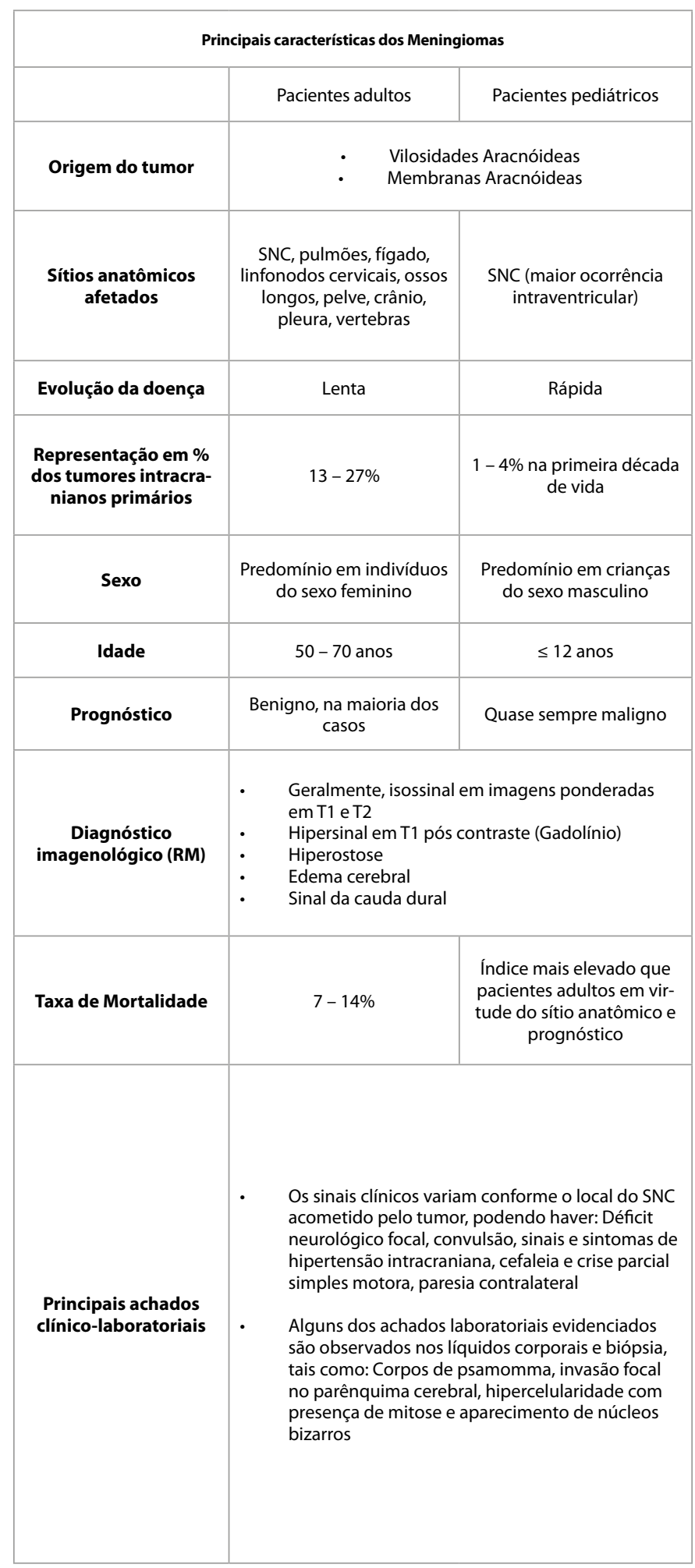

Fonte: Hou et al. (2017)3; Torres et al. (1996)5; Lin et al. (2014)9; Yao et al. (2016) ${ }^{12}$; Figueiredo et al. (1998) ${ }^{14}$; Hanel et al. (2001) Mazzola (2009) ${ }^{16,17}$; Gasparetto et al. (2007) ${ }^{18}$. 


\section{Manifestações clínicas}

Em $90 \%$ dos casos os meningiomas são tumores benignos, sendo o segundo grupo de maior importância dentre as neoplasias primárias que acometem o $\mathrm{SNC}^{19}$.

Os sinais e sintomas provocados pelo meningioma refletem a sua localização e devido ao crescimento lento podem atingir grandes proporções antes de manifestarem algum sinal ou sintoma. Meningiomas da foice e parassagitais do terço médio do seio sagital são os mais frequentes e geralmente se manifestam com paresia contralateral ${ }^{5}$.

Em pacientes pediátricos com suturas não fundidas, pode haver aumento do perímetro cefálico. É descrito ainda déficit neurológico focal, convulsão, sinais e sintomas de hipertensão intracraniana, cefaleia e crise parcial simples motora ${ }^{15}$.

\section{Epidemiologia}

Na literatura, há poucos estudos com dados epidemiológicos para os meningiomas. No estudo de Mendes et al. (2014), quanto ao tipo histológico, os meningiomas transicionais são os mais prevalentes, com $(39,8 \%)$ dos 196 casos de meningiomas, seguido por atípico $(29,1 \%)$, meningotelial (17,3\%), fibroblástico $(7,1 \%)$, angiomatoso (1,5\%), microcístico, clear cells e anaplásico ( $1 \%$ cada) e, psamomatoso e secretor $(0,5 \% \text { cada })^{20}$.

Diversos estudos apontam que esse tipo de tumor acomete principalmente indivíduos do sexo feminino com idade entre 50 e 70 anos. Apresenta incidência de 6 casos por 100.000 habitantes por ano, nos Estados Unidos e prevalência de 97,5 casos por 100.000 habitantes $^{5,19,21}$.

Estudo realizado que avaliou alguns casos de meningiomas pediátricos foram observados alguns aspectos, como o sítio anatômico do tumor e o prognóstico, que diferem do que é comumente observado em indivíduos adultos. Em 1961, uma revisão de 1760 meningiomas evidenciou apenas 19 casos na infância, sendo abordada por alguns autores sua maior incidência em crianças do sexo masculino e menor frequência de aderência à dura-máter. Observou-se ainda diferença entre os sítios anatômicos, com maior ocorrência intraventricular, crescimento rápido, predisposição à transformação sarcomatosa e pior prognóstico ${ }^{15}$.

\section{Ressonância Magnética (RM)}

A imagem por RM é uma técnica em ascendência, utilizada no diagnóstico clínico e na sondagem de aspectos anátomofuncionais. Sua aquisição é decorrente da interação física entre um campo magnético e ondas de radiofrequência gerados pelo equipamento e os prótons de hidrogênio presentes no corpo do indivíduo submetido ao método. Foi descrita em 1946 por Bloch e Purcell, porém, as primeiras imagens obtidas foram possíveis apenas em 1976, evidenciando sua complexidade ${ }^{16,17}$.

A RM produz imagens de maneira não invasiva, em três dimensões, com ampla resolução espacial e de contraste, sem utilizar radiações ionizantes22. Esse exame permite a observação estrutural e dinâmica da matéria por meio de manifestação física entre dois sistemas distintos. Tem seu princípio fundamentado na interação de radiofrequências eletromagnéticas com os núcleos dos átomos de hidrogênio (spin nuclear) submetidos a esse campo, causando excitação atômica e estimulando a captação desses sinais por uma antena, convertendo-os em imagem ${ }^{23}$.

Spin nuclear ou momento angular são propriedades apresentadas por prótons e nêutrons, que representam movimento de rotação sobre seu próprio eixo. Além disso, o próton apresenta um momento em que se comporta como um magneto em consequência de sua carga elétrica, que somado ao movimento é referido como um dipolo magnético, que não somente produz um campo eletromagnético, mas responde à presença de outras fontes ${ }^{23,24}$.

É de suma importância o isolamento do campo magnético para efetivação do princípio da RM, evitando a dispersão dos spins e a perda do sinal ${ }^{22}$.

\section{Sinal da RM e princípios para a aquisição das imagens}

A depender da imagem de RM, muitos órgãos emitem baixos sinais e quando são acometidos por algum tipo de tumor tornam-se mais evidenciados, especialmente após o uso do contraste, em caso de imagens ponderadas em $\mathrm{T} 1^{22}$. Esses sinais podem ser diferenciados como hipointenso, isointenso e hiperintenso ${ }^{18}$.

O sinal é produzido, na ressonância magnética, pelo momento magnético do próton. Trata-se da indução de uma corrente elétrica induzida em uma bobina receptora pelo momento magnético. Esses prótons devem alinhar-se para serem detectados pela bobina. Em situação normal, os prótons encontram-se dispostos aleatoriamente no corpo, havendo a necessidade de serem posicionados em um campo magnético externo para que os spins se alinhem na mesma direção do campo magnético, e no mesmo sentido do vetor ou sentido contrário a ele ${ }^{23}$.

Os efeitos de relaxação T1 (spin-rede) e T2 (spin-spin) dão melhor resolução do contraste de partes moles na RM quando comparada à Tomografia Computadorizada (TC). A água e os lipídeos presentes no organismo são dotados de prótons, possibilitando observar o tempo de relaxação e o comportamento do contraste. Em imagens ponderadas em T1, os líquidos aparecem com hipossinal enquanto nas imagens ponderadas em T2 e DP o sinal dos líquidos é hiperintenso ${ }^{23}$. 


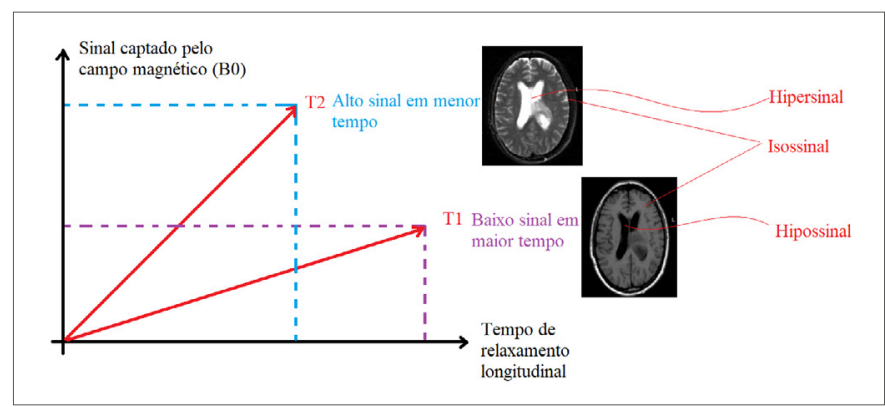

Fonte: Elaborada pelos autores. As imagens de RM estão disponíveis em: [https://pt.slideshare.net/herculys/sequncias-depulso-na-rm].

Quando aplicado no tecido humano, os pulsos de radiofrequência deslocam a direção de magnetização dos prótons de hidrogênio, e posteriormente, ao serem desligados, os spins retornam paulatinamente à sua origem num intervalo denominado tempo de relaxação, sendo diferenciados entre T1 (spin-rede: relacionado ao retorno da magnetização com influência da interação entre os spins e as moléculas de hidrogênio) e T2 (spin-spin: na comunicação dos prótons com campos de outros núcleos) ${ }^{24}$.

Os campos magnéticos induzem a propagação de correntes elétricas, com baixa intensidade, direcionadas de modo a não causarem lesões celulares por meio da quebra de ligações químicas, tornando o método não ionizante, porém, a elevada exposição à esses campos pode causar lesões a partir da geração de radicais livres endógenos ou interação com os canais de cálcio ${ }^{25}$.

Embora a RM convencional colabore bastante para o diagnóstico dos meningiomas, ela não pode determinar o seu grau patológico ou o potencial de crescimento tumoral ${ }^{26}$.

\section{O uso de contrastes na RM}

As técnicas imagenológicas geralmente utilizam substâncias químicas que servem como marcadores, estimulando a produção do sinal da imagem. Tais substâncias são conhecidas como contrastes, que podem ter administração endovenosa ou oral, principalmente ${ }^{22}$.

Grande parte dos compostos utilizados desde 1980 como contraste na RM são à base de quelatos do íon paramagnético gadolínio. Este contraste pode ser comercializado em duas categorias: extracelular inespecífico e intracelular específico, diferenciando-se apenas na molécula quelante carreadora. É encontrado na natureza como um elemento "terras raras", sendo extraído de rochas. Apresenta-se como um cristal branco prateado, com propriedades ferromagnéticas em temperatura ambiente. A aquisição de imagens por ressonância magnética com uso deste contraste intravenoso tem como finalidade evidenciar seu efeito paramagnético sobre os tecidos adjacentes. Na prática, o gadolínio reduz os tempos de relaxamento do hidrogênio ligado à água em ponderação $\mathrm{T} 1$, aumentando o sinal de tecidos biológicos vascularizados. Apresenta fraca afinidade por tecidos biológicos, diferentemente dos contrastes iodados utilizados na TC.

Além disso, o gadolínio é descrito como um contraste mais seguro, porque o volume administrado é bem menor em relação ao da TC, variando entre 10 e $20 \mathrm{~mL}$. Entretanto, podem haver algumas complicações devido ao seu uso, como efeitos adversos, que são descritos com uma incidência de 2-4\%, podendo ocorrer raramente laringoespasmo e choque anafilático. Complicações crônicas podem ser observadas, como a fibrose nefrogênica sistêmica ${ }^{27}$.

\section{Diagnóstico dos meningiomas por RM}

O diagnóstico por imagem, utilizando-se a RM, fornece imagens tridimensionais capazes de diagnosticar meningiomas de forma não invasiva com grande confiança e delinear com precisão as localizações desses tumores em relação às suas estruturas adjacentes para colaborar com o planejamento terapêutico ${ }^{12,19}$.

Meningiomas assintomáticos podem ser identificados e seu crescimento monitorado ao longo do tempo. Além disso, a imagem serve rotineiramente como uma ferramenta essencial para pesquisar a extensão do tumor em vários estágios durante o curso do tratamento, desse modo fornecendo orientações sobre sua eficácia ou a necessidade de novas intervenções ${ }^{12,19,23,28}$. O quadro 2 apresenta os principais benefícios da RM no diagnóstico e monitoramento de meningiomas.

Quadro 2. Principais benefícios da Ressonância Magnética (RM) no diagnóstico e monitoramento de meningiomas.

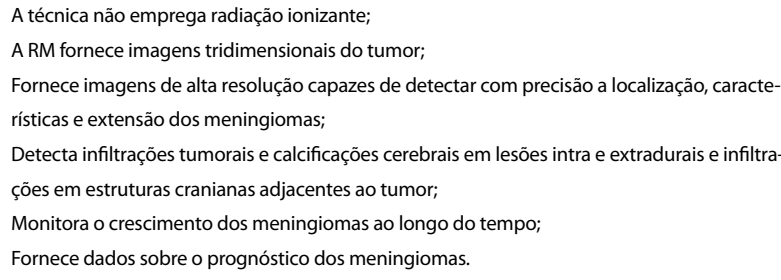

Fonte: Yao et al. (2016)12; Mazzola (2009)16,17; Gasparetto et al. (2007) 18. 
Segundo a Sociedade Americana de Câncer, a biópsia dos espécimes para avaliação molecular e os exames de imagem podem ser bastante úteis no diagnóstico e acompanhamento evolutivo do estadiamento de diversos tipos de tumores, podendo se realizar a Ressonância Magnética para orientação do procedimento invasivo. Além do diagnóstico por imagem, pode-se realizar ainda exames laboratoriais como o Hemograma, EAS e rotina de líquor em busca de possíveis alterações nos líquidos corporais avaliados por cada um destes testes, sendo respectivamente, o sangue, urina e líquido cefalorraquidiano ${ }^{29}$.

Usualmente o crescimento dos meningiomas é lento e apresenta poucos sinais e sintomas no estágio inicial. Meningiomas cerebrais são detectados ao atingirem uma fase avançada com tamanho relativamente grande, capaz de comprimir estruturas adjacentes ${ }^{19}$. O meningioma é o tumor primário intracraniano mais comum, representando cerca de $14-20 \%$ dos casos. Na grande maioria são lesões intradurais, e apenas 1-2\% são extradurais ${ }^{28}$.

Em RM, os meningiomas são geralmente isointensos em T1 eT2, com variações ligadas à textura mais ou menos compacta ou hidratada do tecido, presença de cistos, calcificações ou metaplasia adiposa. Como exemplo, meningiomas microcísticos são ricos em líquido intercelular e dão hipossinal em T1 e hipersinal em T2. Meningiomas psamomatosos densamente calcificados dão hipossinal em T1 e T2. Com contraste, meningiomas mostram captação forte na RM ponderada em T1 (Figura 1) $^{12,19}$.

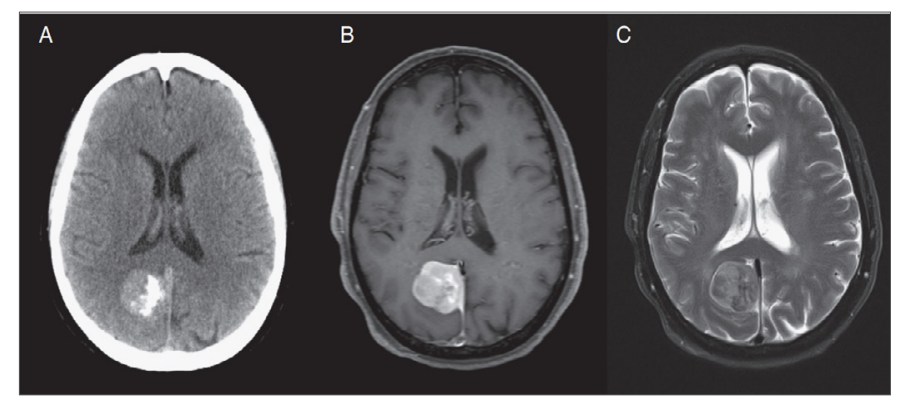

Figura 1. (A) TC sem contraste, (B) RM ponderada em T1 com gadolínio e (C) RM ponderada em T2 de um meningioma falcino direito parcialmente calcificado. Fonte: Adaptada de Huang et al. (2019) ${ }^{30}$.

Segundo estudo realizado em São Paulo-SP-Brasil, os meningiomas na ressonância magnética tem variedade no sinal, na maior parte das vezes isointenso ou hipointenso na sequência T1 e hiperintenso em T2. Contudo, lesões apresen- tam sinal heterogêneo em T1 com destaques para imagens em T2. Com a administração do gadolínio observa-se um realce intenso dessa estrutura. Esse estudo demonstrou prevalência de baixo sinal em 11 na maioria dos tumores (60\%), com maior emissão de sinal em T2 (68\%) e Fluid-attenuated inversion recovery-FLAIR (69\%), com intenso sinal ao uso de contraste, com $26 \%$ dos casos com infiltração óssea e $59 \%$ deles com sinal da cauda dural (Figura 2) ${ }^{18}$.

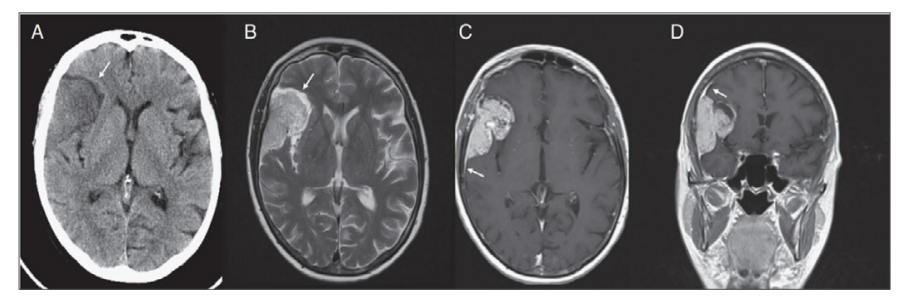

Figura 2. (A) A TC sem contraste mostra uma massa extra-axial com uma fenda de LCR de baixa densidade (seta) entre a massa e o lobo frontal direito adjacente. (B) Imagem de ressonância magnética ponderada em T2 mostra uma massa de sinal intermediário com fenda de LCR (seta). Ressonância magnética aprimorada com gadolínio em planos (C) axial e (D) coronal demonstra realce ávido dentro da massa e um sinal de cauda dural (ponta de seta). Fonte: Adaptada de Huang et al. (2019)30.

Partindo do princípio das imagens ponderadas em T1 e $\mathrm{T} 2$, podemos evidenciar a presença de grande quantidade de sangue nos meningiomas. Isso deve-se à sua hipervascularização, demonstrando maior evidenciação nas imagens ponderadas em $\mathrm{T}^{13,23}$.

O chamado sinal da cauda dural é amplamente conhecido como um achado útil para o diagnóstico de meningioma. Essa estrutura refere-se a um segmento espessado da dura-máter adjacente a uma lesão, destacando-se com o formato de cauda. A impregnação por contraste da dura-máter nas proximidades de meningiomas é atribuída à maior vascularização da camada interna da dura-máter, amplificada pela circulação sanguínea gerada pelo tumor. Esse sinal já foi altamente específico nos meningiomas, mas pode ocorrer também em outros tumores intra ou extra-axiais. Nos meningiomas, apresenta baixa especificidade e boa sensibilidade, podendo variar entre 50$80 \%$. Pode representar alterações vasculares isoladas, invasão tumoral, crescimento tumoral adjacente e não contíguo e micronódulos tumorais ${ }^{13,31}$.

Acredita-se que achados histopatológicos correspondem ao aumento da vascularização e ao realce intramedular, 
refletindo a congestão no osso e ocasionando a hiperintensidade em $\mathrm{T} 2^{13}$.

Tumores hiperintensos em T2 são mais prováveis de serem angioblástico, meningotelial ou sincicial e, também tendem a ser tumores mais suaves. Os tumores hiperintensos em relação à substância cinzenta cerebral em T2, geralmente, são mais propensos a demonstrar componentes de atipia, invasão, angioblástica ou melanocítica. Os tumores hipointensos em T2 são, principalmente, do subtipo fibroblástico, caracterizado por uma matriz colágena densa, e tendem a ser mais firmes. Há uma correlação entre maior conteúdo de colágeno e hipointensidade em T2, e entre maior conteúdo de água e vascularidade e hiperintensidade em $\mathrm{T}^{12}$.

Portanto, achados relevantes no diagnóstico de meningiomas por RM são: vaso intramedular proeminente, realce intramedular e hiperintensidade $\mathrm{T} 2$ intramedular podem ser reconhecidos no crânio adjacente aos meningiomas. Esses achados aparecem tão frequentemente ou mais frequentemente do que hiperostose ${ }^{13}$.

Meningiomas podem provocar hiperostose se infiltrando em ossos do crânio estimulando a proliferação de osteoblastos com deposição de matriz óssea e mineralização, levando ao espessamento do osso afetado (Figura 3) ${ }^{12,13}$.

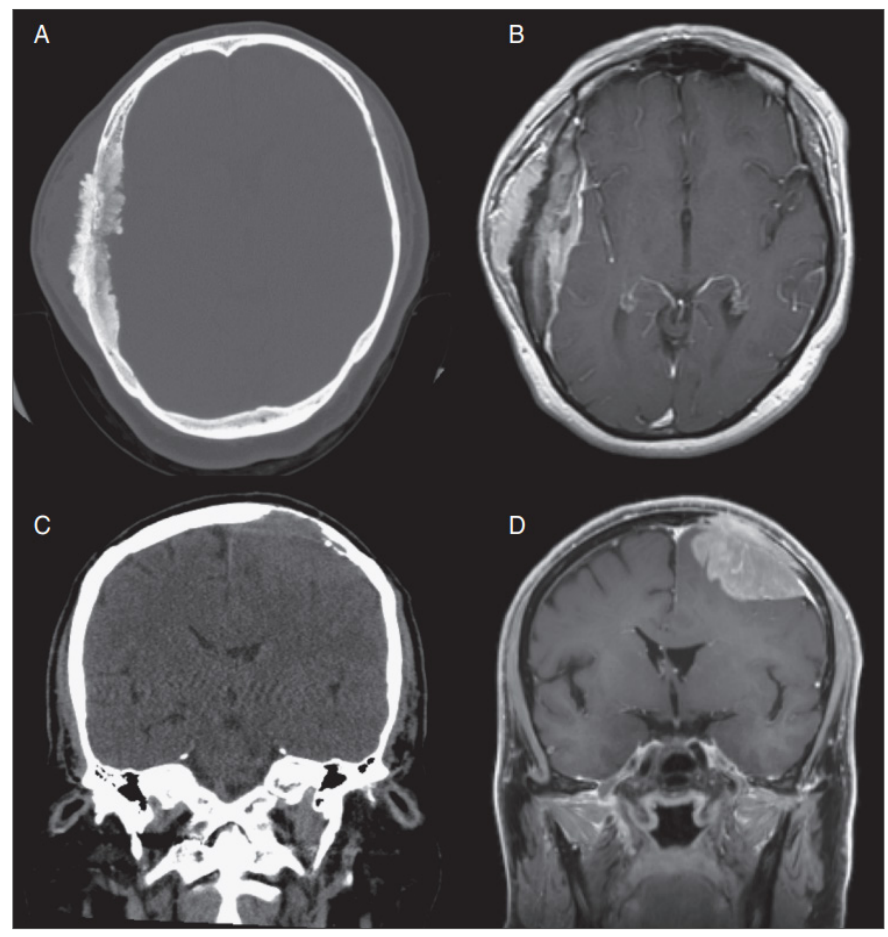

Figura 3. TC sem contraste e ressonância magnética ponderada em T1 com gadolínio demonstrando $(A, B)$ um meningioma grau I com hiperostose profunda e tumor de realce extracalvarial e (C, D) um meningioma de grau III com erosão lítica do osso adjacente da invasão do tumor. Fonte: Adaptada de Huang et al. (2019)30.

Essas evidências encontradas nos casos de meningiomas apresentam mecanismos desconhecidos, mas, acredita-se que estão relacionados entre si, associando-se à hipervascularização observada. Esses achados podem ainda estar diretamente associados ao tamanho do tumor, sendo que, quanto maior o meningioma, maior a vascularização ${ }^{13}$.

\section{Achados histopatológicos}

As características histológicas patognomônicas de um meningioma são formações esféricas de células meningoteliais, chamadas verticilos, que eventualmente se mineralizam em corpos de psammoma ${ }^{32}$.

Desde a publicação das diretrizes revisadas da OMS em 2016, os meningiomas são classificados como grau II de tumores atípicos se eles tiverem 4+ mitoses por dez campos de microscopia óptica consecutivos de alta potência (usando uma objetiva de 40x) ou invasão cerebral, definida como meningioma infiltrando para o córtex subjacente sem uma camada intermediária de tecido conjuntivo"11.

Em estudo em lâminas histopatológicas de 120 tecidos parafinados e corados com HE, determinou a diferenciação de tumores cerebrais de acordo com os critérios da OMS9. Alguns dos achados histopatológicos relacionados aos meningiomas são apresentados na Figura 4.

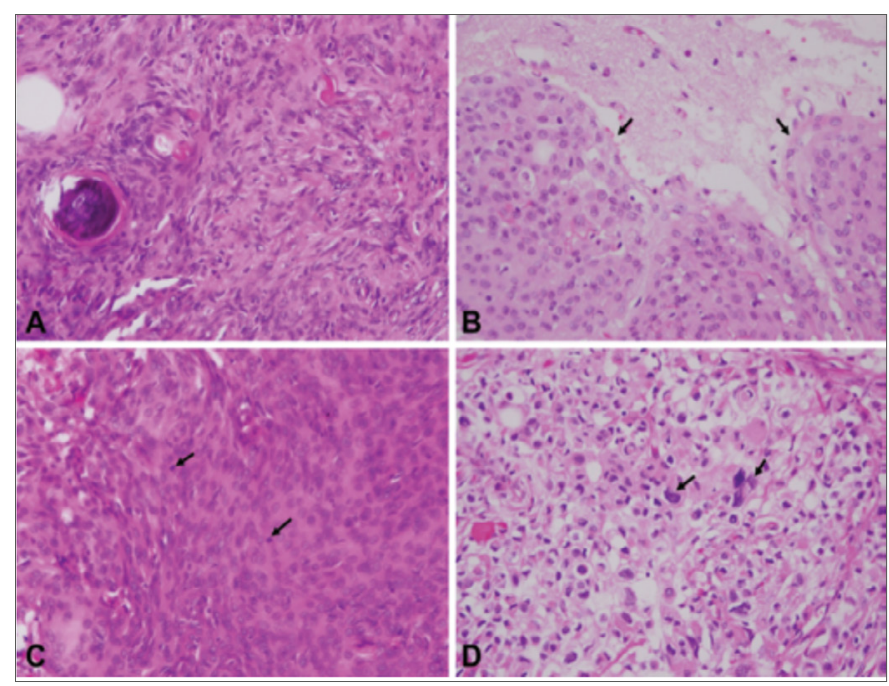

Figura 4. Adaptada de Lin et al. (2014) ${ }^{9}$. Correlação entre a graduação da ressonância magnética e a graduação patológica no meningioma. A. Meningioma meningoteliomatoso (Grau l) 
apresentara corpos de psammoma; B. Meningioma com histopatologia benigna demonstrou invasão focal no parênquima cerebral (Grau II); C. Os meningiomas atípicos (Grau II) mostrou hipercelularidade com presença de mitoses. D. Meningioma anaplásico (Grau III) indicou núcleos bizarros.

\section{Acompanhamento Evolutivo dos Meningiomas por Ressonância Magnética}

Desde seu aparecimento, o meningioma deve ser devidamente diagnosticado e acompanhado em sua progressão como medida de segurança para um tratamento eficaz, necessitando de uma avaliação por imagem mesmo após a ressecção cirúrgica total, sendo associado a uma menor recorrência e maior sobrevida global. Meningiomas residuais são avaliados com maior frequência pela ressonância magnética contrastada, realizada em um prazo de 24 a 72 horas pós operatória. Pode haver um realce dos tecidos de granulação no local, dificultando a diferenciação do tumor residual.

Entretanto, as alterações reativas tendem a ser lineares enquanto o resíduo tumoral apresenta-se de forma nodular. Com o aumento da taxa de recorrência, é recomendado que se realize uma margem ampla de ressecção óssea e da duramáter, podendo haver a complementação deste procedimento com técnicas de radiocirurgia e com o uso de hidroxiuréia em meningiomas benignos irressecáveis e recorrentes, os quais demonstram bastante eficácia, diferente dos meningiomas malignos e atípicos ${ }^{30,33}$.

Há grande susceptibilidade de localizar artefatos pósoperatório imediato devido a presença de ar e hemoderivados na região da ressecção, obscurecendo os locais do tumor realçado, recomendando-se técnicas de eco gradiente $3 \mathrm{D}$ e supressão de gordura, monitorando a proliferação tumoral ao longo do tempo. É possível fazer uma distinção através da RM entre o efeito do tumor, já que a necrose de radiação (que pode ser vista após tratamento de radioterapia) é evidenciada com realce intra-axial. Já nos meningiomas recorrentes, observa-se o espessamento dural ou realce nodular.

A avaliação por imagem é de extrema importância para o acompanhamento evolutivo do meningioma, sendo fundamental que haja comparações entre as imagens nos diversos estágios do tumor para evidenciar sua progressão ou regressão, bem como a observação de seu efeito sobre tecidos peritumorais ${ }^{30}$.

Embora haja um acompanhamento rotineiro dos meningiomas após a ressecção, não existe um consenso estabelecido que se refere à frequência do acompanhamento por imagem. A National Comprehensive Cancer Network fornece uma di- retriz para o acompanhamento dos meningiomas de graus I e Il da OMS em 3, 6 e 12 meses após a cirurgia ou exposição à radiação. Após este período, a cada 6-12 meses por 5 anos e em seguida a cada 1-3 anos. Pode haver uma recorrência tardia desses meningiomas por volta de 10 a 20 anos após o tratamento, sendo fundamental que haja uma vigilância de longo prazo em pacientes jovens ${ }^{30}$.

\section{Conclusão}

Os meningiomas são tumores quase sempre benignos, que acometem o SNC e podem apresentar diferentes características ao longo de sua evolução de acordo com a classificação da OMS em graus que variam de 1 a 3. A importância do diagnóstico por imagem, especialmente da RM, no tratamento contemporâneo e no manejo do meningioma está em sua capacidade de informar a sua localização, crescimento ao longo do tempo e efeito sobre os tecidos adjacentes.

Os meningiomas intracranianos geralmente apresentam-se isointensos em T1 e T2, com algumas variações. Nota-se ainda hipersinal em T1 pós contraste. Além disso, edema cerebral, sinal da cauda dural e infiltração óssea são os achados associados mais frequentes. Entretanto, sua precisão e reprodutibilidade precisam ser validados dentro de populações de pacientes bem definidas, subtipos de tumor e modalidades de tratamento. Com o acompanhamento evolutivo, é possível estimar a taxa de sobrevida dos pacientes diagnosticados com meningiomas e tratados, sobretudo quando são submetidos à ressecção do tumor e que realizam um acompanhamento periódico a fim de identificar possíveis recorrências, que acontecem na maioria dos casos.

\section{Referências}

1. Talamoni A. Anatomia, ensino e entretenimento. Os Nervos e os ossos do ofício uma análise etnológica da aula Anat. Published online 2014:23-37.

2. Madureira LCA, Oliveira CS, Seixas C, Nardi V, Araújo RPC, Alves C. Importância da imagem por ressonância magnética nos estudos dos processos interativos dos órgãos e sistemas. Rev Ciências Médicas e Biológicas. 2010;9(1):13-19.

3. Hou W, Ma Y, Xing H, Yin Y. Imaging characteristics and surgical treatment of invasive meningioma. Oncol Lett. 2017;13(5):2965-2970.

4. Costa Jr LB da, Morais JV de, Lemos S. Meningioma com transformação rabdóide: relato de caso. Arq Neuropsiquiatr. 2003;61(2A):277-280.

5. Torres LFB, Madalozzo LE, Werner B, et al. Meningiomas: estudo epidemiológico e anátomo-patológico de 304 casos. Arq Neuropsiquiatr. 1996;54(4):549-556. 
6. Watts J, Box G, Galvin A, Brotchie P, Trost N, Sutherland T. Magnetic resonance imaging of meningiomas: a pictorial review. Insights Imaging. 2014;5:113-122.

7. Rockhill J, Mrugala M, Chamberlain MC. Intracranial meningiomas: an overview of diagnosis and treatment. Neurosurg Focus. 2007;23(4: E1):1-7.

8. Rubin AS, Pellegrin LG, Porto N da S, Geyer G. Múltiplas metástases pulmonares de meningioma benigno. J Bras Pneumol. 2005;31(2):177-80.

9. Lin BJ, Chou KN, Kao HW, et al. Correlation between magnetic resonance imaging grading and pathological grading in meningioma. J Neurosurg. Published online 2014:1-8.

10. $\mathrm{Cao} \mathrm{H}$, Jiang B, Zhao Y, Fan C. A rare subtype of meningioma. Medicine (Baltimore). 2018;97(23):e11019;1-7.

11. Louis DN, Perry A, Reifenberger G, et al. The 2016 World Health Organization Classification of Tumors of the Central Nervous System: a summary. Acta Neuropathol. 2016;131(6):803-20.

12. Yao A, Pain M, Balchandani P, Shrivastava RK. Can MRI predict meningioma consistency?: a correlation with tumor pathology and systematic review. Neurosurg Rev. Published online 2016:745-53.

13. Matsushima S, Shimizu T, Fukasawa N, Ojiri H. Novel Characteristic Skull Magnetic Resonance Imaging Features Associated With Meningioma. J Comput Assist Tomogr. 2019;43(5):708-12.

14. Figueiredo EG, Aguiar PH, Córdoba BF, Fujimoto $\mathrm{Y}$, Rotta JM, Marino RJ. Meningiomas Supratentoriais Diagnóstico, resultados cirúrgicos e complicações. Arq Neuropsiquiatr. 1998;56(3A):429-35.

15. Hanel RA, Tatsui CE, Araujo JC, et al. Meningiomas em pacientes pediátricos - Relato de 2 casos. Arq Neuropsiquiatr. 2001;59(3A):623-7.

16. Mazzola AA. Ressonancia magnética: princípios de formação da imagem e aplicações em imagem funcional. Rev Bras Física Médica. 2009;3(1):117-29.

17. Mazzola A. Física da RMN. Published online 2009:1-123.

18. Gasparetto EL, Leite CDC, Lucato LT, et al. Intracranial meningiomas: Magnetic resonance imaging findings in 78 cases. Arq Neuropsiquiatr. 2007;65(3A):610-4.

19. Pimentel FL, Guerra GG, dos Santos K, et al. Estudo do perfil epidemiológico dos pacientes com meningioma cerebral da região do Alto Tietê. Rev Neurociencias. 2015;23(3):337-41.

20. Mendes GA, Ongaratti BR, Pereira-Lima JFS. Epidemiologia de uma série de tumores primários do sistema nervoso central. Arq Bras Neurocir. 2014;33(4):279-83.
21. Claus EB, Bondy ML, Schildkraut JM, Wiemels JL, Wrensch M, Black PM. Epidemiology of intracranial meningioma. Neurosurgery. 2005;57(6):1088-95.

22. Guimaraes MD, Schuch A, Hochhegger B, Gross JL, Chojniak R, Marchiori E. Ressonância magnética funcional na oncologia: Estado da arte. Radiol Bras. 2014;47(2):101-11.

23. Hage MCFNS, Iwasaki M. Imagem por ressonância magnética: Princípios básicos. Cienc Rural. 2009;39(4):1287-95.

24. Nunes TCL, da Silveira JB. Ressonância Magnética Nuclear: A física envolvida no diagnóstico por imagens. Rev Conex Eletrônica. 2017;14(1):439-451.

25. Marcilio I, Habermann M, Gouveia N. Campos magnéticos de frequência extremamente baixa e efeitos na saúde: revisão da literatura. Rev Bras Epidemiol. 2009;12(2):105-23.

26. Alegro M de C. Segmentação de tumores do encéfalo em imagens por ressonância magnética baseada em informações texturais. Published online 2009:1-115.

27. Elias JJ, Dos Santos AC, Koenigkam-Santos M, Nogueira-Barbosa MH, Muglia VF. Complicações do uso intravenoso de agentes de contraste à base de gadolínio para ressonância magnética. Radiol Bras. 2008;41(4):263-7.

28. Zakhari N, Torres C, Castillo M, Nguyen TB. Uncommon cranial meningioma: key imaging features on conventional and advanced imaging. Clin Neuroradiol. 2017;27:135-144.

29. American Cancer Society. Brain and spinal cord tumor in adults early detection, Diagnosis, and Staging. Published online 2020:1-19. https://www.cancer.org/ content/dam/CRC/PDF/Public/8569.00.pdf

30. Huang RY, Bi WL, Griffith B, et al. Imaging and diagnostic advances for intracranial meningiomas. Neuro Oncol. 2019;21(S1):i44-i61.

31. Barra FR, Gonçalves FG, Matos V de lima, et al. Sinais em neurorradiologia - Parte 2. Radiol Bras. 2011;44(2):129-33.

32. Menke JR, Raleigh DR, Gown AM, Thomas S, Perry A, Tihan T. Somatostatin receptor $2 a$ is a more sensitive diagnostic marker of meningioma than epithelial membrane antigen. Acta Neuropathol. 2015;130(3):441-3.

33. Barboza EDS, Panagopoulos AT, Lancellotti CLP, Veiga JCE. Meningeoma pós-radioterapia. Arq Neuropsiquiatr. 2006;64(3B):794-7. 